\title{
A Mathematical Model for Control and Optimization of Industrial Rotary Alumina Kiln Process
}

\author{
WEI PENG, RENE V. MAYORGA \\ Faculty of Engineering and Applied Science \\ University of Regina, \\ 3737 Wascana Parkway, Regina, Saskatchewan, S4S 0A2, \\ CANADA
}

\begin{abstract}
Temperature is a crucial factor for clinker quality in the Industrial Rotary Alumina Kiln Process (IRAKP). However, the characteristic of the high temperature, complex kinetics, multivariable, non-linear reaction kinetics, long-time delayed reaction and various raw materials make it difficult to accurately control the temperature in IRAKP through an existing control technology. This paper proposes a dual-responsesurface-based process control (DRSPC) system for the IRAKP in a novel manner. In the DRSPC, instead of the more precise and complicated nonlinear equations, the dual response surface models are fitted to describe the reaction kinetics in the IRAKP and track their standard deviations for stable operation purpose. Because a simultaneous consideration of multiple control targets could address the problem of unstable operation in kilns; the objectives of the DRSPC study are designed as optimizing product quality, minimizing energy consumption and temperature fluctuations. Therefore, the proposed DRSPC goals are to achieve a uniform quality clinker, a high fuel efficiency, and a long refractory life. A weight optimization approach is used to handle these multiple objective functions. The proposed DRSPC can estimate the working conditions of a kiln and predict some optimal manipulated variables to the control system in each control time interval for improving the efficiency of IRAKP. The DRSPC is applied to a real IRAKP for demonstrating its applicability and advantages.
\end{abstract}

Keywords: Rotary alumina kiln, Process control, Optimization, Dual response surface model.

Received: April 29, 2020. Revised: August 16, 2020. Accepted: September 1, 2020. Published: September 10, 2020.

\section{Introduction}

Industrial Rotary Alumina Kiln is a large-scale sintering equipment that broadly applied in cement, metallurgical, chemical, and environmental protection industries. Because of the complicated kinetics, multi-variable characters, nonlinear reaction dynamics, longdelayed reactions and various raw material feed features, in a rotary kiln process is naturally hard to be simulated and modeled [1-3]. So far "there is no mathematical model that adequately reflected the process. and the product quality of IRAKP is usually measured after the clinker cooling down" [3]. Long-delayed reactions seriously affect the online control, and consequently has an effect on the product quality. It is known that the product quality also is greatly influenced by kiln temperatures, especially the maximum sintering temperature [4]. But the measurement of this maximum temperature is extremely difficult due to the high combustion temperature (up to $1500{ }^{\circ} \mathrm{C}$ ), heavy dusty environment, and large temperature fluctuation in the rotary kiln. Hence, only a few successful industrial applications of the kiln process control are reported on the open literatures $[3,5,6]$.

The current control technologies for the IRAKP include proportional integral-derivative (PID) techniques, Artificial Intelligent (AI) methods, model predictive controls (MPC), as well as some hybrid algorithms. The contributions of these technologies include product quality improvement, operational stabilization, cost reduction, and pollution minimization [7]. The PID control is the most common technology used in industrial kiln applications [4, 8], but it usually works well in a stable condition. In the kiln process control, for example, it has a slow response to a process 
upset or frequently uncontrolled disturbances [4]. The artificial intelligent control includes fuzzy and neural-network techniques. It has become a research focus of the kiln process control and has been applied in the industrial field $[2,9,10]$. The AI control requires a prior operational knowledge that highly depends on expert experiences, but the prior operational knowledge may not exist [7,

11]. The model predictive control technology has been greatly developed in recent decades [12-15], successfully applied in industrial kilns to cope with the usual dynamic working conditions. However, its capability still needs be increased, especially for the rapid response to some abnormal problems in kilns. In summary, research needs on the process control of rotary kilns are still substantial.

This paper introduces a distinctive control technology, namely Dual-Response-Surfacebased Process Control (DRSPC), to the prectical applications of the IRAKP. The primary objective of the DRSPC is to optimize product quality. To achieve this objective, the values of major factors should be controlled within allowable ranges. These factors are called controlled variables. For example, the maximum sintering temperature is a key controlled variable in kiln operation. In this DRSPC, Response Surface Methodology (RSM) is used to approximate the relationships of responses (control variables) and input variables (auxiliary measurements and manipulated variables), and its consequence to search for an optimal set of input variables and to optimize the responses by using a set of designed experiments [16-18].

It is worth to mention that the response surface models above are mean quadratic empirical models. In order to maintain the optimal operation in kilns, the standard deviations of responses are considered, and used to build standard deviation quadratic empirical models. A Dual Response Surface Methodology (DRSM) is used in the DRSPC to optimize one of two kinds of responses subjected to an appropriate constraint given by the other [1922]. Obviously, DRSM is derived from RSM that can be used to find an optimal set of input variables and to optimize the responses in kiln operation. The DRSPC only contains quadratic nonlinear models rather than other high-order (more than second order) nonlinear models, so that the optimal solutions can be easily and quickly obtained by a commercial solver because of fast-convergence ${ }^{[23]}$. Therefore, the DRSPC model can overcome the disadvantage of the PID control in industrial kilns to rapidly respond to a process upset or frequently uncontrolled disturbances. Since the DRSPC uses empirical models for control and optimization purposes, it can also go over the deficiencies of AI control that require expert experience. Moreover, due to the empirical models characteristics the various working conditions and associated optimal control operations of an industrial kiln in the previous months, the DRSPC can quickly respond to numerous unusual instabilities that may not be solved by a model predictive control in kilns. However, the accuracy of a quadratic empirical model would be quite low if it had a large number of variables. In practice, a DRSM method is generally limited to a maximum of seven or eight variables due to the curse of dimensionality[24]. So, it is necessary to carefully select variables before we fit an empirical dataset into DRSM models if the number of variables were more than eight.

An application is conducted to demonstrate the proposed DRSPC. At the very beginning of this application, logging was made every sampling interval of an IRAKP. The recorded data sets are used to fit the response surface models that providing an insight into the dynamics and nonlinearity of the kiln. By applying the DRSPC the forecasting of the kiln's work condition and optimal manipulated parameters at each control time interval are generated.

This paper is structured as follows. Section 2 describes the kiln process. Section 3 presents the development of the DRSPC system and its advantages. Section 4 demonstrates an application of the DRSPC. Section 5 presents the conclusion.

\section{Description of IRAKP}

An industrial rotary alumina kiln is a direct contract heat exchanger. It is a long refractory cylindrical vessel with a slight incline from 
horizontal which slowly rotates around its own axis" [7] (as shown in Fig. 1). "The process inside of a rotary kiln includes drying, preheating, decomposing, burning (sintering), and cooling. During the process, raw material slurry is fed at the elevated feeding end of the kiln and it flows down to the discharge end of the rotary kiln due to the kiln's inclination and rotation. Meanwhile, pulverized coal is sprayed from a

burner-pipe

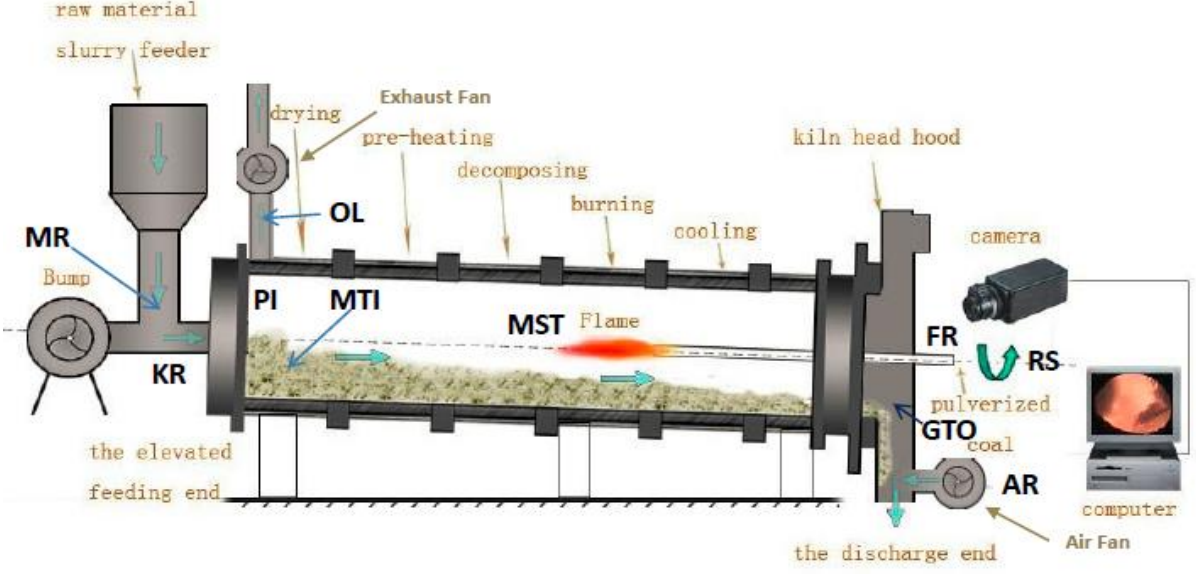

Figure 1. Sketch of an industrial rotary alumina kiln

with the primary air in the burning zone. Thus, heat energy supplied by the combustion of pulverized coal is countercurrent transferred to the raw material slurry in the kiln. Finally, the processed material called clinker is cooled and discharged. The clinker quality is customarily classified into three categories: over-sintered, under-sintered, and normal-sintered" [7]. Since the reactions of the kiln process are very complex, and the previous mainstream control methods have the aforementioned deficiencies (discussed in Section 1), an innovative DualResponse-Surface-based Process Control (DRSPC) system is developed to approximately estimate the dynamic behavior of a real IRAKP and to determine optimal sets of manipulated parameters.

\section{Methodology}

The development of the proposed DRSPC system has following three major steps: (1) Data Acquisition, includes data classification, data range determination, and experimental design. (2) Fitting, fits the dual response surface models. (3) Optimization, builds a dual response surface based optimization and determine manipulated variables [7].

\subsection{Data Acquisition}

The difficulties of the IRAKP control are to find the significant factors that can stablize the temperature fluctuation, and determine their ranges. These imporant factors will be used to design an experiment of the IRAKP with applying the DRSPC. Data acquisition is the necessary step to identify the significant factors and find their ranges. Data acquisition is further used for the experiment design of the IRAKP to achive the objectives of the DSRPC, which inculde optimizing product quality (the primary objective), minimizing temperature fluctuations, as well as minimizing energy consumption.

\subsubsection{Data classification and data rang determination}

The ultimate goal of IRAKP control is to produce high-quality sintered alumina. Here, the quality indexes of sintered alumina are leaching rate and particle size which are measured from the clinker outputs, and will be described in Section 4 regarding an application. It was found that the product quality of sintered alumina is greatly influenced by the maximum sintering temperature [4, 25]. Hence, the maximum sintering temperature (MST) becomes the primary online indicator to product quality. It is called the controlled variable in the DSRPC. 
The MST is a continuous value. It is reported that if MST is above the upper bound of allowable range, the "over-burning" of clinker will be occurred; if MST is below the lower bound of allowable range, the "less-burning" of clinker can be happened [25]. Both "over-

kiln operation. Hence, increasing the allowable range of MST can improve product quality and efficiency of the kiln. It is found that the range of the MST is directly affected by the composition of the raw material $[25,27]$, and the most important impact factor is the aluminasilica. Table 2 demonstrates effects of the alumina-silica ratio of the raw material on the range of MST and product quality. It shows that the higher the ratio of alumina and silica the larger the allowable range of MST. It notes that an IRAKP usually uses a fixed bauxite mine, the composition of the raw material is fixed. Of course, if the composition of the raw material was changed, the parameters of system would be updated correspondingly.

MST is also affected by the following parameters: the fuel flow rate (FR), air flow rate (AR), kiln feed rate (KR), temperature at the kiln outlet (GTO), kiln rotation speed (RS), thermal retention time (TRT) of material, and raw material temperature at the kiln inlet (MTI) [25, 27]. Here, MTI is the most important of the above parameters [25, 27]. A constant MTI helps to maintain a reasonable thermal environment, ensures drying capacity, and preheat the raw materials during the operation of the rotary kiln. However, it is impossible to control MTI to a constant value. The alternative method is to maintain MTI in an allowable burning" and "less-burning" products are poor quality that has low leaching percentage. Only MST fluctuates within an allowable range, the kiln can produce qualified products. Table 1 demonstrates this allowable range of MST in a real industrial rotary alumina

range. Table 1 shows the range of MTI in a real operation of an IRAKP. Meanwhile, it is reported that MTI is majorly influenced by MST, kiln feed rate (KR), pressure at the kiln inlet (PI), RS, moisture content of the raw material (MR), FR, and AR [25-27].

Yi [25] found that the operation of a rotary kiln with high FR, high AR and high RS can produce high-quality products. Moreover, because the thermal retention time (TRT) is controlled by the kiln rotation speed (RS) [25], the parameter of RS will be used to replace the pareameter of TRT in the proposed model.

In summary, MST and MTI are two main paramters that will be used as response variables in the propused DRSPC. They will be controled in allowable ranges during the IRAKP. The aforementioned parameters can be divided into the following two categories: (1) response variables or control variables, including MST and MTI, and (2) independent variables or input variables, containing kiln feed rate (KR), fuel flow rate (FR), air flow rate (AR), kiln rotation speed (RS), pressure at the kiln inlet (PI), gas temperature at the kiln outlet (GTO), and moisture content of the raw material (MR). These parameters are called as independent variables and response variables in the DRSPC, but

they

Table 1. Statistical results of the selected parameters [25]

\begin{tabular}{|c|c|c|c|c|c|c|c|c|}
\hline Name & $\begin{array}{c}\text { MST } \\
\left({ }^{\circ} \mathrm{C}\right)\end{array}$ & $\begin{array}{c}\text { MTI } \\
\left({ }^{\circ} \mathrm{C}\right)\end{array}$ & $\begin{array}{c}\text { GTO } \\
\left({ }^{\circ} \mathrm{C}\right)\end{array}$ & $\begin{array}{c}\text { FR } \\
(\mathrm{RPM})\end{array}$ & $\begin{array}{c}\text { KR } \\
(\mathrm{RPM})\end{array}$ & $\begin{array}{c}\Delta \mathrm{FR} \\
(\mathrm{RPM})\end{array}$ & $\begin{array}{c}\Delta \mathrm{KR} \\
(\mathrm{RPM})\end{array}$ \\
\hline Max & 1250 & 308 & 710 & 1386 & 691 & 79 & 83 \\
\hline Min & 1055 & 230 & 478 & 1114 & 494 & -94 & -136 \\
\hline Averag & 1174 & 263 & 570 & 1269 & 600 & 0 & 0 \\
\hline StdDev & 21.8 & 11.9 & 35.6 & 29.2 & 38.9 & 15.5 & 14.6 \\
\hline
\end{tabular}

MST : maximum sintering temperature, MTI: raw material temperature at kiln inlet, GTO: gas temperature at kiln outlet, FR: fuel flow rate, KR: kiln feed rate, $\triangle \mathrm{FR}$ : change of the fuel flow rate, $\Delta \mathrm{KR}$ : change of the kiln feed rate.

Table 2. Effects of the alumina-silica ratio [25]

\begin{tabular}{|l|l|l|l|l|l|l|}
\hline $\mathrm{A} / \mathrm{S}$ & {$[\mathrm{N} / \mathrm{R}]$} & {$[\mathrm{C} / \mathrm{S}]$} & {$[\mathrm{F} / \mathrm{A}]$} & Mean of & Range of & Leaching percentage (\%) \\
\hline
\end{tabular}




\begin{tabular}{|c|c|c|c|c|c|c|c|}
\hline & & & & $\mathrm{MST}\left({ }^{\circ} \mathrm{C}\right)$ & $\mathrm{MST}\left({ }^{\circ} \mathrm{C}\right)$ & $\eta_{A}$ & $\eta_{N}$ \\
\hline 2.47 & 0.92 & 2.07 & 0.20 & 1180 & $1160-1200$ & 93.04 & 93.46 \\
\hline 2.68 & 0.99 & 2.11 & 0.20 & 1185 & $1160-1210$ & 93.57 & 94.05 \\
\hline 2.96 & 0.96 & 2.10 & 0.20 & 1190 & $1160-1220$ & 94.80 & 93.26 \\
\hline
\end{tabular}

$\mathrm{A} / \mathrm{S}=$ weight ratio of alumina and silica, $\eta_{A}=$ leaching percentage of alumina

$[\mathrm{N} / \mathrm{R}]=$ molecular ratio of sodium oxide and (alumina + iron oxide),

$[\mathrm{C} / \mathrm{S}]=$ molecular ratio of calcium oxide and silica,

$[\mathrm{F} / \mathrm{A}]=$ molecular ratio of iron oxide and alumina, $\eta_{N}=$ leaching percentage of sodium oxide.

are called as input variables and control variables in the IRAKP. In practical applications, the input variables are also named as "auxiliary measurements". Moreover, some of the above input variables are manipulatable, e.g. FR, AR, KR, and RS. We are interested in the changes of these manipulatable variables, and denoted them by $\Delta \mathrm{FR}, \Delta \mathrm{AR}, \Delta \mathrm{KR}$ and $\Delta \mathrm{RS}$. They are called "manipulated variables" in the IRAKP.

All the above parameters will be measured and collected through the actual IRAKP, which is used to construct a dataset. The details of data acquisition are described in Section 4.2. Their measurement locations are shown in Figure 1.

\subsubsection{Experimental design}

Before designing the experiments, the following structures of the industrial rotary kiln are assumed: (1) the kiln process is continuous, and (2) correlations exist among variables.

An experiment is designed as follows: (1) continuous operating an industrial rotary alumina kiln; (2) sampling the work condition parameters at every time interval of $\Delta t$, where $\Delta t$ is the time between two adjacent samples; (3) measuring the product quality by a chemical analysis at every time interval of $\Delta T$, where $\Delta T$ is the time between two adjacent measurements of product quality, and $\Delta T$ is much longer than $\Delta t$.

An appropriate sampling interval of $\Delta t$ is governed by the dynamics of the process which should allow the completion of all data samplings, data analysis, optimization calculation, and control actions. The designed experiments are then used for the development of DRSPC. Since the proposed method is an empirical model whose purpose is to produce satisfactory high quality clinker, this empirical model can only be trained with "correct operation data". The correct operation is the operation that yields high quality clinker in various working conditions. Therefore, the operation data associated with low-quality product is useless and will be removed from the sampling data.

\subsection{Fitting response surface models}

Box and Wilson [29] first applied the Response Surface Method in 1952 to research the relationship between a response and a set of input variables. Vining and Myers ${ }^{[28]}$ fitted two second-order polynomial models for the mean response and standard deviation of responses separately. In their research, the optimization of one of the polynomial models subjected to an appropriate constraint given by the other [31]. A general DRSM model is developed for the industrial rotary kiln as follows:

$$
\begin{aligned}
& y=h_{o}+\boldsymbol{S}^{\prime} \boldsymbol{h}+\boldsymbol{S}^{\prime} \boldsymbol{H} \boldsymbol{S} \\
& \sigma_{y}=g_{o}+\boldsymbol{S}^{\prime} \boldsymbol{g}+\boldsymbol{S}^{\prime} \boldsymbol{G} \boldsymbol{S}
\end{aligned}
$$

where $g_{0}=\alpha_{0}, h_{0}=\beta_{0}, \boldsymbol{g}=\left(\alpha_{1}, \alpha_{2}, \ldots \alpha_{k}\right)^{\prime}$, $\boldsymbol{h}=\left(\beta_{1}, \beta_{2}, \ldots \beta_{k}\right)^{\prime}$, and

$$
\begin{aligned}
& \boldsymbol{H}=\frac{1}{2}\left[\begin{array}{cccc}
2 \beta_{11} & \beta_{12} & \ldots & \beta_{1 k} \\
\beta_{21} & 2 \beta_{22} & \cdots & \\
\vdots & \vdots & \ddots & \vdots \\
\beta_{k 1} & \beta_{k 2} & \cdots & 2 \beta_{k k}
\end{array}\right] \\
& \boldsymbol{G}=\frac{1}{2}\left[\begin{array}{cccc}
2 \alpha_{11} & \alpha_{12} & \ldots & \alpha_{1 k} \\
\alpha_{21} & 2 \alpha_{22} & \ldots & \\
\vdots & \vdots & \ddots & \vdots \\
\alpha_{k 1} & \alpha_{k 2} & \cdots & 2 \alpha_{k k}
\end{array}\right]
\end{aligned}
$$

where $h_{0}, g_{0}, \boldsymbol{h}, \boldsymbol{g}, \boldsymbol{H}$ and $\boldsymbol{G}$ are the appropriate scalars, $(k \times 1)$ vectors, and $(k \times k)$ matrices for the estimated coefficients, respectively; $y$ and $\sigma_{y}$ 
are the mean and standard deviation of the MST and MTI, respectively; $S$ and $S^{\prime}$ are $(k \times l)$ vectors of the input variables and their transpose, respectively.

For the kiln process control, several sets of second-order polynomial models (DRSM models) need to be fitted for controlled variables. They are the MST main model with its standard deviation model, and MTI main model with its standard deviation model. The responses of the above models are denoted as $y_{M S T}$ and $\sigma_{M S T}, y_{M T I}$ and $\sigma_{M T I}$, respectively. The input variables for both main and standard deviation models are same, which include the auxiliary measurements (e.g. $M S T, F R, A R, K R, G T O, R S$ and $M T I$ ) and manipulated variables (e.g. $\triangle F R$, $\triangle A R, \triangle K R$ and $\triangle R S$ ). The manipulated variables here refer to the value changes between two adjacent sampling intervals. For example, $\Delta F R_{i}$ $=F R_{i+1}-F R_{i}$. The responses and input variables in above models correspond to different sampling interval. For example, if the current sampling interval is $i(i=1,2, \ldots, n)$, the input variables stand for the current sampling interval $i$ and the responses denote the next sample interval $i+1$.

\subsection{Dual response surface process control}

Lin and $\mathrm{Tu}$ [19] proposed an objective minimized function, namely the Mean Squared Error (MSE), to find an optimal set of conditions such that the mean response will be close to the target value, while the standard deviation response keeps small. Hence, a dual response surface based optimization can avoid misleading optimum (which the mean response is close to the target value but the standard deviation response is large), and then produce robust results.

In this study, the eventual objective of the DRSPC is to optimize product quality. According to Yi' study [25], a simultaneous consideration of multiple control targets could address the problem of unstable operation. Since the clinker quality is highly dependent on MST, MTI, and GTO, the primary objective can be converted to control these temperatures to their target values of $T_{M S T}, T_{G T O}$, and $T_{M T I}$, respectively. We can further convert the primary objective to minimize the differences between the temperature responses and their target temperatures, $\left(y_{M S T}-T_{M S T}\right),\left(y_{G T O}-T_{G T O}\right)$, and $\left(y_{M T I}-T_{M T I}\right)$. Consequently, the second objective of the DRSPC is to minimize the standard deviations of these temperatures, which are $\sigma_{M S T}$, $\sigma_{G T O}$, and $\sigma_{M T I}$. Since the proposed model is for an IRAKP in China, a large amount of data recorded from this kiln is used for the model building. The analysis of this recorded data shows that MST dominates the clinker quality, and followed by MTI, but GTO has little impact on product quality. Hence, GTO and its standard deviation are deleted from the primary and secondary objectives. Of course, different kilns have different situations, GTO may have an important impact on product quality in other kilns rather than MTI.

We also consider about the minimization of energy consumption, which can be converted minimizing the manipulated variables (e.g. $\triangle F R$, $\triangle A R, \triangle K R$ and $\triangle R S)$. Some manipulated variables are fixed during the operation such as $\triangle R S$, and some manipulated variables are highly correlated with each other such as the manipulation of $\triangle A R$ is dependent on $\triangle F R$. Hence, only $\triangle F R$ and $\triangle K R$ are used in the DRSPC. The DRSPC can be formulated as follows:

$$
\begin{aligned}
\operatorname{Min} f= & w_{1}\left[\left(y_{M S T}-T_{M S T}\right)^{2}+\sigma_{M S T}^{2}\right] \\
& +w_{2}\left[(\Delta F R)^{2}+(\Delta K R)^{2}\right] \\
& +w_{3}\left[\left(y_{M T I}-T_{M T I}\right)^{2}+\sigma_{M T I}^{2}\right]
\end{aligned}
$$

Subject to:

$$
\begin{aligned}
& y=h_{o}+\boldsymbol{S}^{\prime} \boldsymbol{h}+\boldsymbol{S}^{\prime} \boldsymbol{H} \boldsymbol{S} \\
& \sigma_{y}=g_{o}+\boldsymbol{S}^{\prime} \boldsymbol{g}+\boldsymbol{S}^{\prime} \boldsymbol{G} \boldsymbol{S} \\
& y_{\boldsymbol{L}} \leq y \leq y_{\boldsymbol{U}} ; \quad \boldsymbol{S}_{\boldsymbol{L}} \leq \boldsymbol{S} \leq \boldsymbol{S}_{\boldsymbol{U}}
\end{aligned}
$$

where $y$ and $\sigma_{y}$ are the mean and standard deviation responses of the MST and MTI for the future step, respectively; $T_{M S T}$ and $T_{M T I}$ are the targets of the MST and MTI, respectively; $\triangle F R$ and $\Delta K R$ is the predicted change values of the fuel flow rate and kiln feed rate, respectively; $S_{L}$ and $S_{U}$ are the lower and upper bounds of $\boldsymbol{S}$; $w_{1}$ to $w_{3}$ are the weighting factors reflecting different priorities in the calcination process. 
Three weighting factors can be adjusted by the Control Laws that are discussed in the next section. It is worth mentioning that the Pareto Fronts approximation ${ }^{[30]}$ will be applied for the multi-objective optimization in our future work.

\section{Proposed Approach Application}

In this section, the proposed DRSPC is implemented to demonstrate its ability of process control in the complex and dynamic operation of kiln.

\subsection{Overview of the study rotary kiln}

The study object is an $\phi 5 \times 110 m$ industrial rotary alumina kiln that located at the Alumina Corporation of China in Henan province [7]. The operation stages of the IRAKP include "drying, preheating, decomposing, burning, and cooling. The main characteristics in each stage are described as follows: (a) in the drying stage, most water in the raw material slurry is evaporated by gas heat. The temperature of the gas in the drying zone consequently drops from $700 \sim 800^{\circ} \mathrm{C}$ to $180 \sim 250^{\circ} \mathrm{C}$, (b) in the preheating stage, the temperature of the material is increased to $600^{\circ} \mathrm{C}$ and some thermal decomposition reactions are performed to remove crystal water from the raw material, (c) in the decomposing stage, the temperature of the material is increased to $1000^{\circ} \mathrm{C}$, the crystal water is continually decomposed and the carbonate begins to decompose. Meanwhile, some reactions occurred to compose new materials such as $\mathrm{Na}_{2} \mathrm{O} \cdot \mathrm{Fe}_{2} \mathrm{O}_{3}, \quad \mathrm{Na}_{2} \mathrm{O} \cdot \mathrm{Al}_{2} \mathrm{O}_{3}$, and $\mathrm{Na}_{2} \mathrm{O} \cdot \mathrm{Al}_{2} \mathrm{O}_{3} \cdot 2 \mathrm{SiO}_{2}$, (d) in the burning (sintering) stage, the temperature of the material is increased to $1200 \sim 1300^{\circ} \mathrm{C}$, and the actual temperature of the gas reaches up to $1500^{\circ} \mathrm{C}^{\prime \prime}[7]$. The sintering procedure is described by the following chemical equations:

$$
\begin{aligned}
& \mathrm{Na}_{2} \mathrm{O} \cdot \mathrm{Al}_{2} \mathrm{O}_{3} \cdot 2 \mathrm{SiO}_{2}+4 \mathrm{CaO} \\
& =\mathrm{Na}_{2} \mathrm{O} \cdot \mathrm{Al}_{2} \mathrm{O}_{3}+2\left(2 \mathrm{CaO} \cdot \mathrm{SiO}_{2}\right) \\
& 2 \mathrm{CaO}+\mathrm{SiO}_{2}=2 \mathrm{CaO} \cdot \mathrm{SiO}_{2} \\
& \mathrm{CaO}+\mathrm{TiO}_{2}=\mathrm{CaO} \cdot \mathrm{TiO}_{2}
\end{aligned}
$$

Final one (e) is the cooling stage, which the processed material, called clinker, is cooled and discharged. The temperature of the gas in this stage is $400 \sim 600^{\circ} \mathrm{C}$.

The studying kiln was manually controlled with low productivity and unstable product quality, which is required to be upgraded. The DRSPC was proposed to this IRAKP starting from the parameter sampling.

\subsection{Sampling}

The working condition parameters of the IRAKP were continually sampled from January 23, 2008 to April 27, 2008 [7]. The sampling interval of $\Delta t$ was set as 5.5 minutes. A total of 28,067 sets of parameters were recorded. Each set of parameters includes the maximum sintering temperature (MST), raw material temperature at kiln inlet (MTI), gas temperature at the kiln outlet (GTO), kiln feed rate (KR), fuel flow rate (FR), air flow rate (AR), kiln rotation speed (RS), and pressure at the kiln inlet (PI), etc. Fig. 2 shows three temperatures of the MST, MTI and GTO. However, the temperature cannot directly be measured because temperature sensors are not available along the rotary kiln. Hence, several indirect indicators are used to estimate the temperature, which include the colorimeter signal and flame image process. The flame image process is that use a camera to take images of flame, and then convert the images into temperature values through an image processing algorithm [7].

The quality of the alumina was measured from the clinker outputs and was performed every 0.5 -hour interval $\Delta T$. High quality alumina is porous with a bulk density of 1.2 to $1.45 \mathrm{~kg}$ / L. According to the results of product quality, four periods (shown in Table 3) were selected for building the DRSPC model, which includes 2,400 sets of operational parameters and kiln working condition data. The production during these four periods meets the quality requirement (normal-sintered alumina). The idea of the proposed approach is to train the mathematical models by the dataset of operational parameters and kiln working conditions, and then use these trained models in the control process of the rotary kiln. Here, the valuable data sets should be collected from the correct operations that can produce satisfactory quality products in a variety 
of different working conditions (including unstable and long-delayed reactions) to ensure that the proposed empirical model is properly trained.

Table 3 Four periods selection for building the DRSPC model

\begin{tabular}{|c|c|c|c|}
\hline Period 1 & Period 2 & Period 3 & Period 4 \\
\hline Jan. 26, & Feb. 15, & Mar. 6, & Apr. 9, \\
2008 & $2008 \sim$ & $2008 \sim$ & $2008 \sim$ \\
Jan. 29, & Feb. 18, & Mar. 8, & Apr. 11, \\
2008 & 2008 & 2008 & 2008 \\
\hline
\end{tabular}

\subsection{Dual response surface models}

MST and MTI with their standard deviations are chosen as the responses (controlled variables) for the dual response surface models. Because most of manipulated variables are fixed (or allowed a very small fluctuation) in the real operation of the kiln [25-28], these fixed variables are considered as constants and will be removed from the quadratic equations. Therefore, only two manipulated variables of $\triangle K R$ and $\triangle F R$ are used

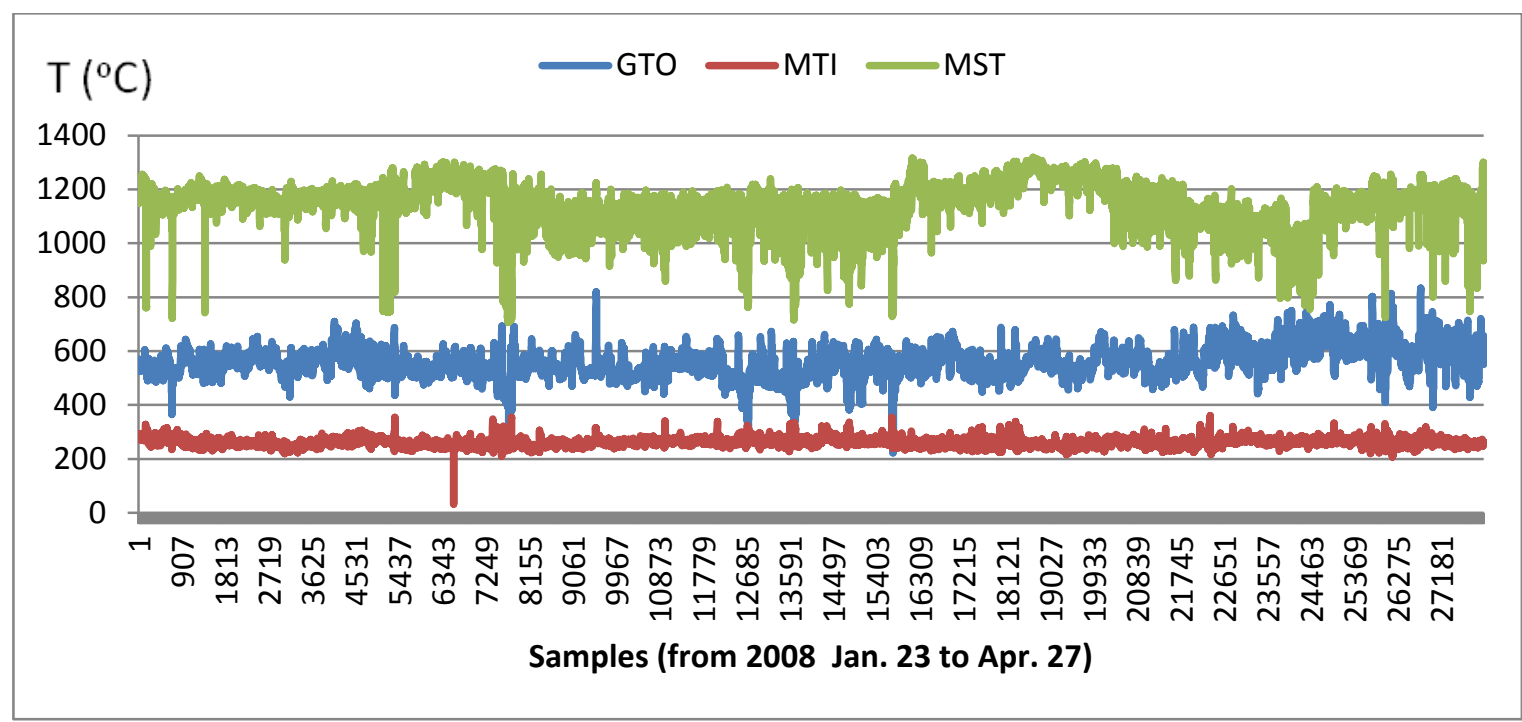

Figure 2. Temperatures of the IRAKP

in the dual response surface models. Further, theoretically the control of the fuel-air ratio is very important to combustion efficiency. Due to the temperature of the kiln having a small fluctuation in a normal working condition of the kiln, a fixed fuel-air ratio is enough to provide good combustion efficiency [33].

According to the developed model fitting methods, the responses of the MST main ( $\left.y_{M S T}\right)$ and their standard deviation $\left(\sigma_{M S T}\right)$ are at the next sampling instant; the auxiliary measurements (MST, FR, KR, GTO and MTI) and manipulated variables $(\triangle F R$ and $\triangle K R)$ are at the current sampling instant. Similarly, the responses of the MTI main $\left(y_{M T I}\right)$ and standard deviation $\left(\sigma_{M T I}\right)$ are at the next sampling instant; the auxiliary measurements $(M T I, F R, K R$ and $M S T)$ and the manipulated variables $(\triangle F R$ and $\triangle K R)$ are at the current sampling instant. The dual response surface models are fitted by the
MATLAB function "rstool" using the selected data in a confidence level of $\alpha=0.05$, and shown as follows:

$$
\begin{aligned}
& 10^{4} \cdot y_{M S T}= \\
& -6621551-1225 \cdot G T O+38595 \cdot M T I+9661 \cdot M S T \\
& +2703 \cdot F R+46803 \cdot \Delta F R+3557 \cdot K R-4003 \cdot \Delta K R \\
& -0.72 \cdot G T O \cdot M T I+0.39 \cdot G T O \cdot M S T+3.84 \cdot G T O \cdot F R \\
& +8.93 \cdot G T O \cdot \Delta F R+0.27 \cdot G T O \cdot K R-6.45 \cdot G T O \cdot \Delta K R \\
& -35.09 \cdot M T I \cdot M S T+4.87 \cdot M T I \cdot F R-55.56 \cdot M T I \cdot \Delta F R \\
& -10.11 \cdot M T I \cdot K R-23.51 \cdot M T I \cdot \Delta K R-18.19 \cdot M S T \cdot F R \\
& +8.77 \cdot M S T \cdot \Delta F R-2.08 \cdot M S T \cdot K R-10.06 \cdot M S T \cdot \Delta K R \\
& -36.21 \cdot F R \cdot \Delta F R+3.86 \cdot F R \cdot K R+20.17 \cdot F R \cdot \Delta K R \\
& +5.34 \cdot \Delta F R \cdot K R+19.81 \cdot \Delta F R \cdot \Delta K R-1.94 \cdot K R \cdot \Delta K R \\
& -3.7 \cdot G T O^{2}+5.7 \cdot M T I^{2}+13.57 \cdot M S T^{2}+5.36 \cdot F R^{2} \\
& -26.12 \cdot \Delta F R^{2}-3.14 \cdot K R^{2}+5.28 \cdot \Delta K R^{2}
\end{aligned}
$$$$
R^{2}=0.994
$$ 
$+9485740+1027 \cdot G T O-4459 \cdot M T I-12546 \cdot M S T$

$-6 \cdot F R-901 \cdot \Delta F R-5992 \cdot K R-7333 \cdot \Delta K R$

$+4.2 \cdot G T O \cdot M T I-3.83 \cdot G T O \cdot M S T-0.7 \cdot G T O \cdot F R$

$-2.69 \cdot G T O \cdot \Delta F R-0.13 \cdot G T O \cdot K R+9.97 \cdot G T O \cdot \Delta K R$

$+10.24 \cdot M T I \cdot M S T-6.9 \cdot M T I \cdot F R-17.12 \cdot M T I \cdot \Delta F R$

$+3.69 \cdot M T I \cdot K R-12.88 \cdot M T I \cdot \Delta K R+2.14 \cdot M S T \cdot F R$

$+5.07 \cdot M S T \cdot \Delta F R-0.29 \cdot M S T \cdot K R-1.24 \cdot M S T \cdot \Delta K R$

$-0.38 \cdot F R \cdot \Delta F R+2.87 \cdot F R \cdot K R+5.27 \cdot F R \cdot \Delta K R$

$+2.61 \cdot \Delta F R \cdot K R-8.96 \cdot \Delta F R \cdot \Delta K R-0.25 \cdot K R \cdot \Delta K R$

$+2.96 \cdot G T O^{2}-5.72 \cdot M T I^{2}+3.87 \cdot M S T^{2}-0.69 \cdot F R^{2}$

$+9.53 \cdot \Delta F R^{2}+1.58 \cdot K R^{2}+5.96 \cdot \Delta K R^{2}$

$R^{2}=0.972$

$10^{4} \cdot y_{M T I}=$

$+2152806+1782 \cdot M T I-133 \cdot M S T-2448 \cdot F R$

$-1405 \cdot \Delta F R+2012 \cdot K R+6926 \cdot \Delta K R+0.75 \cdot M T I \cdot M S T$

$+4.92 \cdot M T I \cdot F R-3.41 \cdot M T I \cdot \Delta F R+5.06 \cdot M T I \cdot K R$

$-3 \cdot M T I \cdot \triangle K R-2.82 \cdot M S T \cdot F R-0.31 \cdot M S T \cdot \Delta F R$

$+0.06 \cdot M S T \cdot K R+0.78 \cdot M S T \cdot \Delta K R+1.76 \cdot F R \cdot \Delta F R$

$-2.18 \cdot F R \cdot K R-3.64 \cdot F R \cdot \Delta K R+0.91 \cdot \Delta F R \cdot K R$

$-2.57 \cdot \Delta F R \cdot \Delta K R-4.18 \cdot K R \cdot \Delta K R-5.25 \cdot \mathrm{MTI}^{2}$

$+1.54 \cdot M S T^{2}+2.26 \cdot F R^{2}+0.51 \cdot \Delta F R^{2}-0.5 \cdot K R^{2}$

$-4.96 \cdot \Delta K R^{2}$

$R^{2}=0.711$

$10^{4} \cdot \sigma_{M T I}=$

$-254508+1461 \cdot M T I+319 \cdot M S T-228 \cdot F R$

$-600 \cdot \Delta F R+150 \cdot R+931 \cdot \Delta K R-1.55 \cdot M T I \cdot M S T$

$-1.61 \cdot M T I \cdot F R \quad-4.44 \cdot M T I \cdot \Delta F R-0.22 \cdot M T I \cdot K R$

$-5.22 \cdot M T I \cdot \Delta K R-1.93 \cdot M S T \cdot F R+0.31 \cdot M S T \cdot \Delta F R$

$+0.12 \cdot M S T \cdot K R-1.06 \cdot M S T \cdot \Delta K R+1.12 \cdot F R \cdot \Delta F R$

$-0.33 \cdot F R \cdot K R+0.25 \cdot F R \cdot \Delta K R-0.06 \cdot \Delta F R \cdot K R$

$-5.66 \cdot \Delta F R \cdot \Delta K R+2.22 \cdot K R \cdot \Delta K R+5.15 \cdot M T I^{2}$

$+1.09 \cdot \mathrm{MST}^{2}+1.19 \cdot F R^{2}-1.31 \cdot \Delta F R^{2}+0.11 \cdot \mathrm{KR}^{2}$

$-0.42 \cdot \Delta K R^{2}$

$R^{2}=0.659$

where, the term yмsт is the response variable of sintering temperature, it is related on the input variables defined in Equation 9a. We hope that $y_{M S T}$ equals to the target value of $M S T$ which means the kiln will produce high-quality clinker. Similarly, in Equation 10a, the term $y_{M T I}$ is the response variable of the raw material temperature at kiln inlet, it is related on the input variables defined in Equation 10a. We hope that $y_{M T I}$ equals to the target value of MTI which means the kiln will produce high-quality clinker. The units of the MST, MTI, and GTO are Celsius degrees $\left({ }^{\circ} \mathrm{C}\right)$. Because the $\triangle F R$ and $\triangle K R$ are controlled by the flow pumps, the pump speeds (RPM) are used as unit for them. Table 1 lists the statistical results of selected parameters, which include the maximum values, minimum values, averages, and standard deviation values. $R^{2}$ is correlation coefficient to measure the reliability of these fitted quadratic equations.

\subsection{Optimization}

A nonlinear optimization model is generated for the IRAKP based on the DRSPC as follows:

$$
\text { Min } \begin{aligned}
f & =w_{1}\left[\left(y_{M S T}-1174\right)^{2}+\sigma_{M S T}^{2}\right]+w_{2}\left[(\Delta F R)^{2}+(\Delta K R)^{2}\right] \\
& +w_{3}\left[\left(y_{M T I}-263\right)^{2}+\sigma_{M T I}^{2}\right]
\end{aligned}
$$

Subject to:

Equations (9a), (9b), (10a) and (10b)

$0 \leq y_{M S T} \leq 1300 ; \quad 0 \leq y_{M T I} \leq 500 ; \quad-150 \leq \Delta F R \leq$

$150 ; \quad-150 \leq \Delta K R \leq 150$

The weighting coefficients of $w_{1}$ to $w_{3}$ are used to reflect the different priorities in the calcination process. According to the recorded data from an industrial rotary alumina kiln in China, $1174^{\circ} \mathrm{C}$ of MST is the primary goal in the calcination process. Considering about its standard deviation (shown in Table 1), the allowable range of MST is designed as $1174^{\circ} \mathrm{C} \pm$ $20^{\circ} \mathrm{C}$. $263^{\circ} \mathrm{C}$ of MTI is the secondary objective in the calcination process. Considering about its standard deviation (shown in Table 1), the allowable range of MTI is designed as $263^{\circ} \mathrm{C} \pm$ $11^{\circ} \mathrm{C}$. If the values of MST and MTI were out of its range, the product quality would be adversely influenced severely. Hence, the related weights of $w_{1}$ and $w_{3}$ should be increased when the values of MST and MTI are out of their ranges. Thus, several control laws of the weighting values adjustment are designed as follows:

If $\mid$ MST $-1174 \mid \leq 20$, then $\mathrm{w}_{1}=1$;

If $\mid$ MST $-1174 \mid>20$, then $\mathrm{w}_{1}=[(\mathrm{MST}-$

$1174) / 20]^{2}$;

If $\mid$ MTI $-263 \mid \leq 11$, then $\mathrm{w}_{3}=1$;

If $\mid$ MTI $-263 \mid>11$, then $w_{3}=[(M T I-263) / 11]^{2}$. 
The purpose of the control scheme is to stabilize the work condition in kiln, which eventually achieve the goals of uniform quality clinker, high fuel efficiency, and long refractory life. The term "stable kiln work condition" means the conditions in kiln only have very small changes, or no changes at all. An upset condition is caused by large fluctuations in MST and MTI temperatures or frequent large changes in manipulation variables (e.g. FR and KR). So, the purpose of Equation 11a is to minimize the changes of kiln work conditions subject to the search of the optimal solutions in Equation $11 \mathrm{~b}$ and the ranges of $\triangle F R$ and $\triangle K R$.

In Equation 11a, we design the weighting coefficients of $w_{1}$ to $w_{3}$ that are used to enhance the stable kiln work conditions. For example, we assume an upset condition that | MST $1174 \mid>20$, then $w_{l}$ will be increased to [(MST - 1174)/20] ${ }^{2}$ from 1 (Please check the control laws above). Since the objective of Equation 11a is to minimize the summation of three terms, the optimal value of $y_{M S T}$ solved by Equation $11 \mathrm{~b}$ must be closer to the target temperature of $1174^{\circ} \mathrm{C}$ under the effect of the increased value of $w_{1}$, while the optimal value of $\left(\sigma_{\mathrm{MST}}\right)^{2}$ must be smaller. At the same time, Equation $11 \mathrm{~b}$ will generate the optimal results for the manipulation variables to handle this upset and return the kiln to a stable condition.

\subsection{Results and discussion}

A 550-minute sample was collected from IRAKP to verify the functions of DRSPC. The sample contains 100 sub-samples of duration $\Delta t$ with several disturbances. MATLAB software was used for the above optimization of DRSPC. The duration of each optimization is only several seconds that far less than 5.5 minutes of the designed sampling interval of $\Delta t$, which means an operator or auto-controller has enough time to correct the operational errors according to the optimal results from this optimization model in real operating conditions.
Multiple control targets of the MST and MTI are simultaneously considered in the DRSPC. Fig. 3 shows two observed MST curves that obtained from the manual control system and DRSPC system. The curves illustrate that all large deviations of MST in the manual control system are considerably reduced in the DRSPC system, which will contribute to high quality products. The main reason of reducing the deviation of MST may be the DRSPC system subjects to a constraint of minimizing the deviation of MST.

Fig. 4 displays two observed MTI curves obtained from the manual control system and DRSPC system. The curves indicate that all large deviations of MTI in the manual control system are reduced in the DRSPC system, but this reduction is not as good as that of MST curve in Fig. 3. The reason might be: (1) the DRSPC models were regressed from the dataset of previous corrected operations of the IRAKP, and these corrected operations were directly measured based on the value of MST rather than MTI; (2) in this study, most MST are below the target temperature $\left(1174^{\circ} \mathrm{C}\right)$ but most MTI are over the target temperature $\left(263^{\circ} \mathrm{C}\right)$, which results in a control conflict. For example, we need to increase MST while decreasing MTI, where increasing MST requires improvements in FR and KR, but decreasing MTI requires drops in FR and KR; (3) due to the deviations of MST is larger than the deviations of MTI. According to the control laws, the weight value of MST will be greater than that of MTI, which would result in the deviations of the MST have a larger reduction in the DRSPC optimization. As mentioned in the previous section, the product quality of sintered alumina is greatly influenced by the maximum sintering temperature $[4,25]$, which means the less MTI reducation will not affect the product quality.

Various weight ratios are designed to check the optimal sensitivity of weight. Table 4 illustrates the simulation results of varioius weight ratios which can be summarized as:

(1) from the experiments $\# 1, \# 3$, $\# 4$, and $\# 6$, it is 


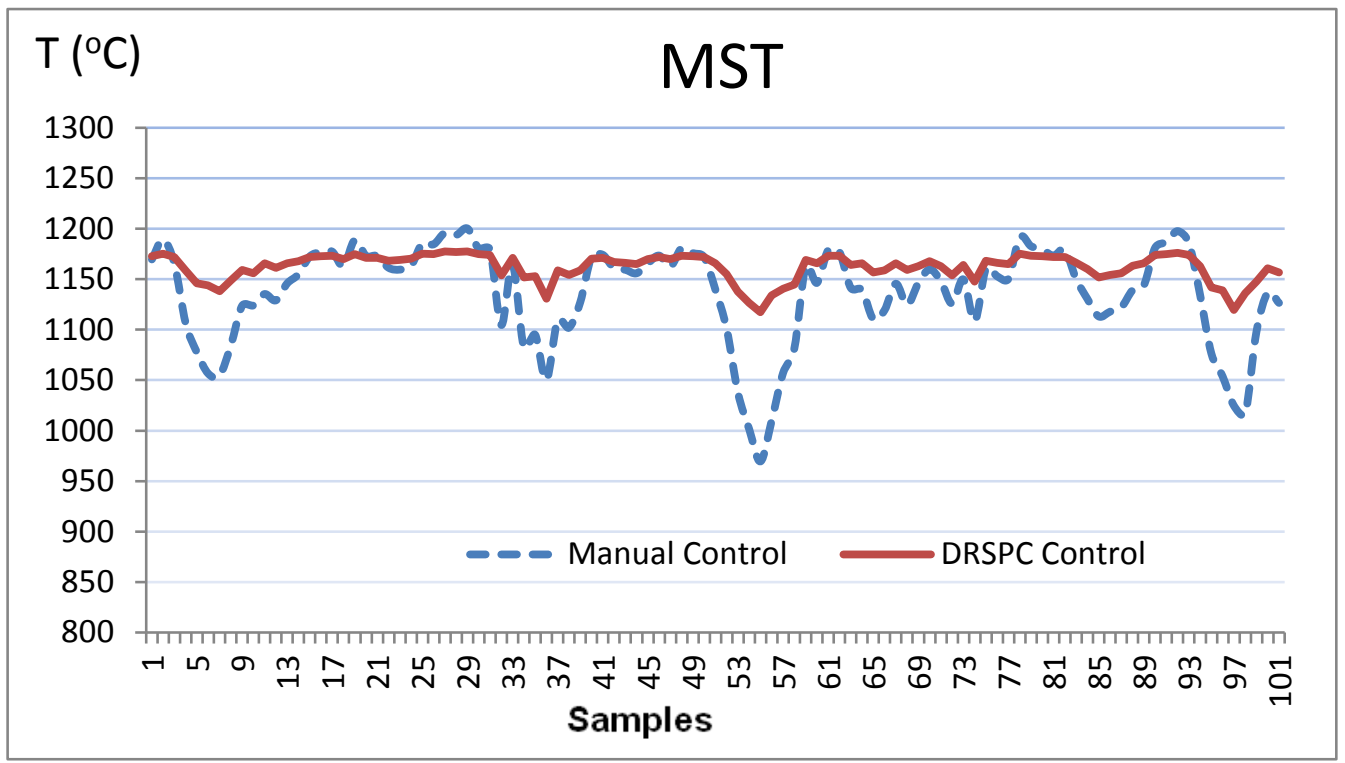

Figure 3. MST results of manual control and DRSPC control

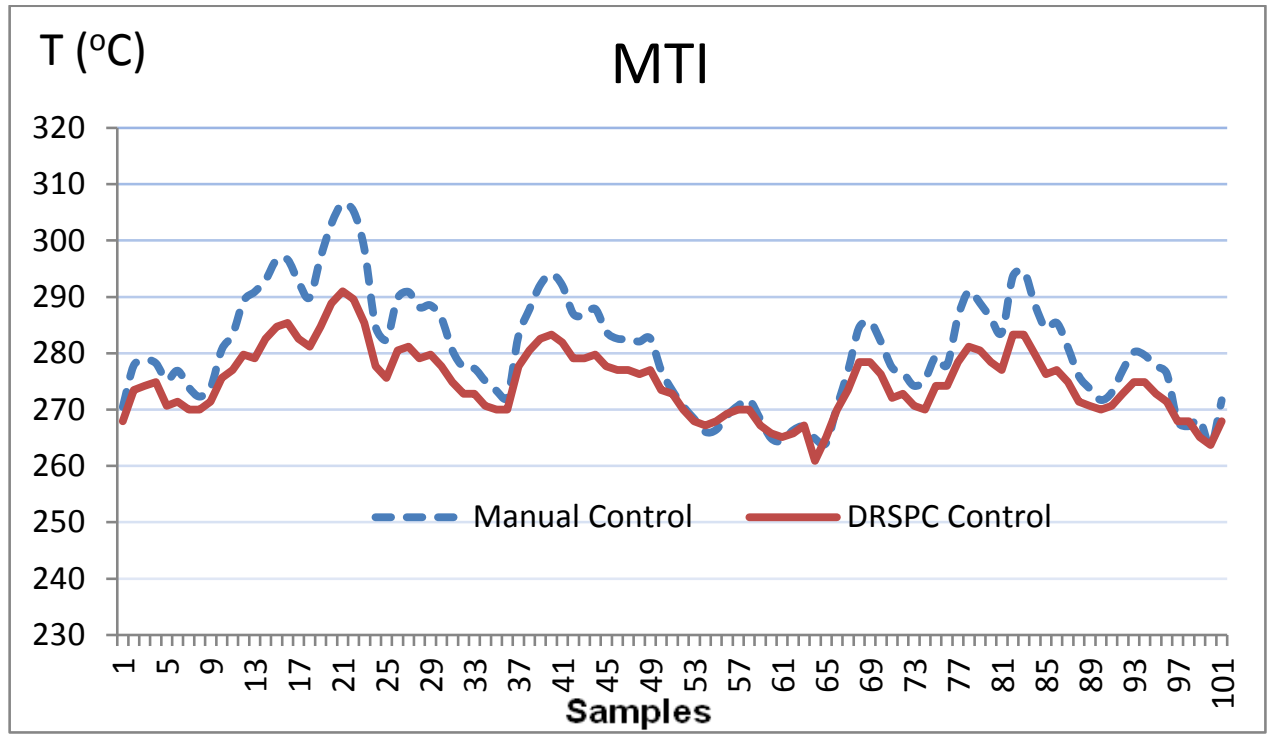

Figure 4. MTI results of manual control and DRSPC control

found the weight of the MST $\left(w_{1}\right)$ can result in more optimization sensitivity than the weight of the MTI $\left(w_{3}\right)$, which means that MST control is more effective than MTI control in improving the quality of sintered alumina;

(2) from experiments \#4 to \#7, it is found that control both of MST and MTI is better than only control of MST in improving the product quality;

(3) from experiments \#7 to \#10, we found that the weight ratio of $\left(w_{1}: w_{2}: w_{3}=100: 1: 100\right)$ is more efficitive than the weight ratio of $\left(w_{1}: w_{2}\right.$ : $\left.w_{3}=1000: 1: 1000\right)$ in optimizing the product quality;
(4) there is a tradeoff between the temperature control and energy consumption.

(5) from the experiment of "ContLaw", it is found that the control laws make considerable contributions to the process control.

Since the rotary kiln studied in this paper is a long refractory cylindrical vessel that takes $40-$ 50 minutes to transport raw material from the feeding end to the sintering zone, the underheated or over-heated materials will consequently effect on the sintering quality in the sintering zone after this $40-50$ minutes transportation. The original manual control system only sets a single sintering temperature 
target for maintaining MST, which could result in an unstable operation

Table 4. Results for variations of the weighted coefficients

\begin{tabular}{|c|c|c|c|c|c|c|c|}
\hline & $w_{1}$ & $w_{2}$ & $w_{3}$ & $\begin{array}{c}\text { MST } \\
\left({ }^{\circ} C\right)\end{array}$ & $\begin{array}{c}\text { MTI } \\
\left({ }^{\circ} C\right)\end{array}$ & $\begin{array}{c}\Delta F R \\
(R P M)\end{array}$ & $\begin{array}{c}\Delta K R \\
(R P M)\end{array}$ \\
\hline Target & - & - & - & 1174 & 263 & 0 & 0 \\
\hline 1 & 0 & 0 & 1 & 1125 & 277 & -137.7 & -150 \\
\hline 2 & 0 & 1 & 0 & 1139 & 295 & 0 & 0 \\
\hline 3 & 0 & 1 & 1 & 1140 & 295 & 0.8 & -0.8 \\
\hline 4 & 1 & 0 & 0 & 1161 & 293 & 61.6 & -29.7 \\
\hline 5 & 1 & 0 & 1 & 1165 & 292 & 45.3 & -54.7 \\
\hline 6 & 1 & 1 & 0 & 1145 & 295 & 10.8 & -6.8 \\
\hline 7 & 1 & 1 & 1 & 1146 & 294.5 & 11.3 & -7.6 \\
\hline 8 & 10 & 1 & 10 & 1158 & 293.1 & 33.5 & -32 \\
\hline 9 & 100 & 1 & 100 & 1165 & 292.1 & 43.3 & -51.4 \\
\hline 10 & 1000 & 1 & 1000 & 1166 & 291.6 & 45.1 & -54.4 \\
\hline ContLaw & 5.76 & 1 & 12.76 & 1157 & 293.2 & 27.4 & -32 \\
\hline
\end{tabular}

Note: 1. the parameters are chosen from the record of 08/1/23 17:33;

2. the original values of parameters are: $\operatorname{MST}\left(1126^{\circ} \mathrm{C}\right), \operatorname{MTI}\left(302.3^{\circ} \mathrm{C}\right)$, GTO $\left(514.6^{\circ} \mathrm{C}\right), \operatorname{FR}(1187.6 \mathrm{RPM}), \mathrm{KR}(539.4 \mathrm{RPM})$.

condition [28]. Our experiments (\#4 to \#7) show that a simultaneous consideration of multiple control targets could address the problem of unstable operation. The developed DRSPC system has two temperature targets for MST $\left(1174^{\circ} \mathrm{C}\right)$ and $\operatorname{MTI}\left(263^{\circ} \mathrm{C}\right)$, respectively. These two control targets resulted in a stable operation, and the temperature of MST was controlled within a narrow range of $1120^{\circ} \mathrm{C}$ to $1180^{\circ} \mathrm{C}$ (shown in Fig. 3). Comparing with the allowable temperature range of $\mathrm{MST}\left(1055^{\circ} \mathrm{C}\right.$ to $\left.1250^{\circ} \mathrm{C}\right)$, the stable temperature of MST can ensure a high quailty of sintered alumina.

In practical applications, some indirect indices were used to estimate the temperature in the burning zone of a rotary kiln such as use flame image method [26]. However, these indirect indices may cause some measurement errors due to the high temperature and heavy dusty environment along the rotary kiln. In this DRSPC, two target temperatue values of MST and MTI are obtained by averaging the values of the recorded temperature, which can statistically eliminate the measurement errors of the temperature. The quailty of sintered alumina is then improved.

Stability is an important issue for a control system [34-36]. The considered rotary alumina kiln obviously is a bounded input bounded output (BIBO) system because the input parameters and the output or response parameters ( $M S T$ and $M T I$ ) are bounded by certain value ranges. The system is stable and controllable if we can determine the suitable input-output control loop pairing [37]. This suitable input-output contol loop pairing can be found because we can obtain expected state values of the auxiliary measurements (MST, FR, $K R, G T O$ and $M T I)$ though operating the inputs of manipulated variables ( $\triangle F R$ and $\triangle K R$ ) based on initial states of the auxiliary measurements; also because we can estimate previous states of the auxiliary measurements (MST, FR, KR, GTO and $M T I$ ) though observing the outputs of responses of MST main $\left(y_{M S T}\right)$ and their standard deviation $\left(\sigma_{M S T}\right)$ which shown in Figure 3. Therefore, the studying IRAKP also is an internal stable system.

\section{Conclusions}

This paper has presented a Dual-ResponseSurface-Based Process Control (DRSPC) system for the practical applications of IRAKP in a novel manner. By applying the DRSPC the forecasting of the kiln's work condition and optimal manipulated parameters at each control time interval are generated. The developed DRSPC has the following properties:

- Multiple objectives include optimizing product quality, minimizing energy 
consumption, and minimizing temperature fluctuations.

- Rapid optimization due to the DRSPC only involves quadratic models.

- Robust results due to the standard deviations of the responses are considered.

- The unstable operation problem is solved by designing multiple control targets of the MST and MTI simultaneously.

The proposed approach has been applied to a real case study of an industrial rotary alumina kiln in China. The control laws are designed for this DRSPC system to make considerable contributions to the kiln process control. The results can provide a solid basis for guiding the real-time process control of industrial rotary alumina kilns.

\section{Acknowledgements}

The authors would like to thank Dr. M. He, and J. Zhang, from the College of Electrical and Information Engineering, Hunan University, Changsha China for providing the data sets.

This paper research has been supported by a grant (No: 155147-2013) from the Natural Sciences and Engineering Research Council of Canada (NSERC).

\section{References}

[1] D. Moles, et al., The aerodynamics of the rotary cement kiln. J. Inst. Fuel. 1973, vol.46, p.353.

[2] M. Jarvensivu, et al., Intelligent Control System of an Industrial Lime Kiln Process, Control Eng. Pract. 2001, vol.9(6), p.589.

[3] A. Kouadri, et al., An adaptive threshold estimation scheme for abrupt changes detection algorithm in a cement rotary kiln. J. Comput. Appl. Math. 2014, vol.259(B), p.835.

[4] P. Mills, et al., A practical study of adaptive control of an alumina calciner, Automatica. 1991, vol.27(3), p.441.

[5] S. Larsson, et al., PIV analysis of merging flow in a simplified model of a rotary kiln, Exp. Fluids. 2012, vol.53(2), p.545.

[6] Z. Yi, et al., Mathematic simulation of heat transfer and operating optimization in alumina rotary kiln, J. Cent. South Univ. 2013, vol.20, p.2775.

[7] W. Peng, Dealing With Uncertainty in Engineering and Management Practices, Ph.D.
Dissertation, University of Regina, Saskatchewan, Canada, 2011, p.181.

[8] C. Hang, K. Sin, Online auto tuning of PID controllers based on the cross correlation technique, IEEE T. Ind. Electron. 1991, vol.38 (6), p.20.

[9] Y. Wang, A Hybrid Intelligent Control for Industrial Rotary Kiln Plant (Chinese), Control Theory and Appl. 1996, vol.13(6), p.770.

[10] W. Chen, et al., GA-based fuzzy neural controller design for municipal incinerators, Fuzzy Set. Syst. 2002, vol.129, p.343.

[11] Y. Huang, et al., IPCS: An integrated process control system for enhanced in-situ bioremediation, Environ. Pollut. 2008, vol.151 (3), p.460.

[12] J. Valiquette, M. Savoie, Practical Aspects of model predictive control implementation on an industrial lime kiln. Tappi Journal. 1999, May, p.130.

[13] R. Zanovello, H. Budman, Model predictive control with soft constraints with application to lime kiln control, Comput. Chem. Eng. 1999, vol.23(6), p.791.

[14] K. Stadler, et al., Model predictive control of a rotary cement kiln, Control Eng. Pract. 2011, vol.19(1), p.1.

[15] R. Teja, et al., Control and optimization of a triple string rotary cement kiln using model predictive control, Int. Federation of Auto. Control. 2016, vol.49(1), p.748.

[16] C. Anderson-Cook, et al., Response surface design evaluation and comparison, J. Stat. Plann. and Infer. 2009, vol.139.

[17] R. Myers, et al., Response surface methodology: a retrospective and literature survey, J. Quality Tech. 2004, vol.36, p.53.

[18] W. Peng, et al., A rapid fuzzy optimization approach to multiple sources water blending problem in water distribution systems, Urban Water J. 2012, vol.9(3), p.177.

[19] D. Lin, W. Tu, Dual response surface optimization, J. Qual. Technol. 1995, vol.27, p.34.

[20] S. Assarzadeh, M. Ghoreishi, A dual response surface-desirability approach to process modeling and optimization of $\mathrm{Al} 2 \mathrm{O} 3$ powder mixed electrical discharge machining (PMEDM) parameters, Int. J. Adv. Manuf. Technol. 2012, vol.64 (9-12), p.1459.

[21] X. Zhou, et al., Ensemble of surrogates for dual response surface modeling in robust parameter design. Qual. Reliab. Eng. Int. 2013, vol.29(2), p.173. 
[22] W. Peng, et al., A robust optimization approach for real-time multiple source drinking water blending problem, J. Water Supply Res. T. 2012, vol.61 (2), p.111.

[23] W. Peng, R. V. Mayorga, Hydraulic reliability assessment and optimal rehabilitation/ upgrading schedule for water distribution systems, Appl. Math.- SCIRP, 2016, vol.7, p. 2336 ..

[24] J. Christensen, C. Bastien, Introduction to Structural Optimization and Its Potential for Development of Vehicle Safety Structures. In book: Nonlinear Optimization of Vehicle Safety Structures, 2016, vol.169.

[25] Z. Yi, Thermal Analysis and Control Study on Alumina Rotary Kiln, Ph.D. Dissertation, Central South University, Changsha, China, 2008, p.17.

[26] C. Lin et al., An alumina rotary kiln monitoring system based on flame image processing, Trans. Inst. Meas. Control, 2018, vol.41(14): 014233121881008.

[27] H. Liu, et al., Numerical simulation of particle motion and heat transfer in a rotary kiln, Powder Technol., 2016, vol.287, p.239.

[28] B. Xu, Research and development of integrated automation system for alumina clinker burning rotary kiln, M.A.Sc Thesis, Northeastern University, Shengyang, China, 2004. p.10.

[29] G. Box, K. Wilson, On the experimental attainment of optimum conditions, J. Roy. Stat. Soc. 1951, vol. B(13), 1.

[30] G. Vining, R. Myers, Combining taguchi and response surface philosophies: a dual response approach, J. Qual. Technol. 1990, vol.22(1), p.38.

[31] W. Peng, R. Mayorga, Developing a stat. model to improve drinking water quality for WDS by minimizing HMR, Water 2018, vol.10(7), p.939.

[32] B. Marjavaara, et al., Hydraulic turbine diffuser shape optimization by multiple surrogate model approximations of Pareto fronts, J. Fluid Eng. 2007, vol.129 (9), p.1228.

[33] X. Zhang, et al., Intelligent predictive control strategy applied to sintering temperature in rotary kiln based on image feedback (Chinese), Control Theory and Appl. 2007, vol.24 (6), p.995.

[34] C. Thammarat, D. Puangdownreong, Design of Fractional Order PID Controller for Induction Motor Speed Control System by Cuckoo Search, Int. J. Circuits, Syst. Signal Process, 2019, vol.13, p.92
[35] T. Jitwang, et al., Optimal PIDA Controller Design for Three-Tank Liquid-Level Control System with Model Uncertainty by Cuckoo Search, Int. J. Circuits, Syst. Signal Process, 2019, vol.13, p. 60.

[36] Honghui Mu, Jun Tang, The Composite Control Method for the GDI Engine Idle Speed Control, Int. J. Circuits, Syst. Signal Process, 2019, vol.13, p. 97.

[37] S. Sunori et al., Control System Design with Dead Time Compensation for a Multivariable Lime Kiln Process, J. Graph. Era Univ., 2017, vol. 5(2), p.147

\section{Symbols Used}

\begin{tabular}{|c|c|l|}
\hline$\alpha$ & {$[-]$} & Element of Matrix \\
\hline$\beta$ & {$[-]$} & Element of Matrix \\
\hline $\mathrm{g}_{0}$ & {$[-]$} & Appropriate scalar \\
\hline $\mathrm{h}_{0}$ & {$[-]$} & Appropriate scalar \\
\hline $\mathbf{g}$ & {$[-]$} & Appropriate vector \\
\hline $\mathbf{h}$ & {$[-]$} & Appropriate vector \\
\hline $\mathbf{G}$ & {$[-]$} & Appropriate matrix \\
\hline $\mathbf{H}$ & {$[-]$} & Appropriate matrix \\
\hline $\mathbf{S}$ & {$[-]$} & $\begin{array}{l}\text { Vectors of the input } \\
\text { variables }\end{array}$ \\
\hline $\mathbf{S}$ & {$[-]$} & $\begin{array}{l}\text { Transpose vectors of the } \\
\text { input variables }\end{array}$ \\
\hline $\mathbf{S}_{\mathbf{L}}$ & {$[-]$} & $\begin{array}{l}\text { Lower bound vectors of the } \\
\text { input variables }\end{array}$ \\
\hline $\mathbf{S}_{\mathbf{U}}$ & {$[-]$} & $\begin{array}{l}\text { Upper bound vectors of the } \\
\text { input variables }\end{array}$ \\
\hline $\mathrm{MST}$ & {$\left[{ }^{\circ} \mathrm{C}\right]$} & $\begin{array}{l}\text { Maximum sintering } \\
\text { temperature }\end{array}$ \\
\hline $\mathrm{MTI}$ & {$\left[{ }^{\circ} \mathrm{C}\right]$} & $\begin{array}{l}\text { Raw material temperature } \\
\text { at the kiln inlet }\end{array}$ \\
\hline$y$ & {$\left[{ }^{\circ} \mathrm{C}\right]$} & Mean of MST and MTI \\
\hline$\sigma_{y}$ & {$\left[{ }^{\circ} \mathrm{C}\right]$} & $\begin{array}{l}\text { Standard deviation of MST } \\
\text { and MTI }\end{array}$ \\
\hline$w$ & {$[-]$} & Weight coefficient \\
\hline $\mathrm{T}_{\mathrm{MST}}$ & {$\left[{ }^{\circ} \mathrm{C}\right]$} & Target temperature of MST \\
\hline $\mathrm{T}_{\mathrm{MTI}}$ & {$\left[{ }^{\circ} \mathrm{C}\right]$} & Target temperature of MTI \\
\hline$\Delta F R$ & {$[\mathrm{kw} / \mathrm{s}]$} & $\begin{array}{l}\text { The change of fuel flow } \\
\text { rate }\end{array}$ \\
\hline$\Delta K R$ & {$[\mathrm{~kg} / \mathrm{s}]$} & The change of kiln feed rate \\
\hline$\Delta t$ & {$[\mathrm{~s}]$} & Sampling interval time \\
\hline$\Delta T$ & {$[\mathrm{~s}]$} & $\begin{array}{l}\text { Product quality measuring } \\
\text { interval time }\end{array}$ \\
\hline
\end{tabular}

\section{Creative Commons Attribution License 4.0 (Attribution 4.0 International, CC BY 4.0)}

This article is published under the terms of the Creative Commons Attribution License 4.0

https://creativecommons.org/licenses/by/4.0/deed.en_US 